\title{
A simple model for DNA denaturation
}

\author{
Thomas Garel ${ }^{1}$, Cécile Monthus ${ }^{1}$ and Henri Orland ${ }^{1,2}$ \\ ${ }^{1}$ CEA, Service de Physique Théorique, 91191 Gif-sur-Yvette, Cedex, France. \\ ${ }^{2}$ Institute for Theoretical Physics, University of California at Santa Barbara, \\ Santa Barbara, CA 93106
}

(November 2, 2018)

Pacs: 87.14.Gg, 05.70.Fh, 63.70.+h, 64.10.+h

\begin{abstract}
Following Poland and Scheraga, we consider a simplified model for the denaturation transition of DNA. The two strands are modeled as interacting polymer chains. The attractive interactions, which mimic the pairing between the four bases, are reduced to a single short range binding term. Furthermore, base-pair misalignments are forbidden, implying that this binding term exists only for corresponding (same curvilinear abscissae) monomers of the two chains. We take into account the excluded volume repulsion between monomers of the two chains, but neglect intra-chain repulsion. We find that the excluded volume term generates an effective repulsive interaction between the chains, which decays as $1 / r^{d-2}$. Due to this long-range repulsion between the chains, the denaturation transition is first order in any dimension, in agreement with previous studies.
\end{abstract}

Submitted for publication to: "Europhys. Lett."

Saclay, SPhT/01-003 
The problem of the denaturation of DNA has been of physical interest for a long time [1],2], and can be roughly characterized as the adsorption (or more accurately, desorption) transition of one strand onto (from) the other. Experiments indeed show that the fraction of bound base pairs exhibits, as a function of temperature, a series of sharp jumps, linked to the local melting of these heterogeneous paired structures. This is in contrast with many theories [1,2], which yield a continuous denaturation transition below four dimensions.

The full problem is rather complicated since different binding energies are associated with different bases contents (such as different AT and GC frequencies).

Moreover, since the denaturation transition decreases the stiffness of a DNA molecule, the backbone elasticity may play an important role in the transition [3]. Along these lines, the helical structure of the molecule has been also considered [4]

To disentangle polymeric from disorder effects, simplifying assumptions have to be made. In this work, we follow a time honored approach where (i) disorder is neglected (homogeneous DNA approximation) (ii) base-pair mismatch is forbidden (pairing is possible only between corresponding monomers along the two chains). (iii) we consider excluded volume interactions only between the two chains.

Experimentally, the chains are not very long $(N \approx 1000)$ and have a fairly large persistence length $\left(l_{p} \sim 20\right.$ pairs $)$. This suggests that for typical distances of the chains smaller than several persistence lengths, excluded volume effects between monomers in the same chain will be negligible compared to excluded volume effects between monomers of the two chains.

Closely related work can be found in references [5,6] where the existence of a first order transition in three dimensions has been clearly linked, via numerical simulations or scaling arguments, to the short range (excluded volume) repulsion between the chains (by first order, we mean a phase transition with a finite latent heat and/or some finite length scales). 
In our model, the partition function of the two chains of length $N$ reads :

$$
\begin{aligned}
Z= & \int \mathcal{D} \vec{r}_{1}(s) \mathcal{D} \vec{r}_{2}(s) \exp \left(-\frac{d}{2 a^{2}} \int_{0}^{N} d s\left(\left(\frac{d \vec{r}_{1}}{d s}\right)^{2}+\left(\frac{d \vec{r}_{2}}{d s}\right)^{2}\right)-\beta \int_{0}^{N} d s v\left(\vec{r}_{1}(s)-\vec{r}_{2}(s)\right)\right) \\
& \times \exp \left(-g \int_{0}^{N} d s \int_{0}^{N} d s^{\prime} \delta\left(\vec{r}_{1}(s)-\vec{r}_{2}\left(s^{\prime}\right)\right)\right)
\end{aligned}
$$

where $d$ is the space dimension, $a$ is the Kuhn length of the monomers, $\beta$ is the inverse temperature, $g$ is the excluded volume parameter and $v\left(\vec{r}_{1}-\vec{r}_{2}\right)$ is the short range binding potential for monomers $s$ of chain 1 and 2. In the following, we will model this interaction by an attractive ( $d$ dimensional) spherical well potential, of radius $r_{0}$ and depth $-V_{0}$.

An approximate treatment of the excluded volume interaction will be given first. Then, we study the unbinding phase transition in the framework of a quantum analogy [7]. We finally compare our results with references [5,6].

It is clear from equation (11), that one should focus on the relative coordinate $\left(\vec{r}_{1}(s)-\right.$ $\left.\vec{r}_{2}(s)\right)$ of the two chain system to study a possible adsorption transition. Introducing the new coordinates

$$
\begin{aligned}
\vec{R}(s) & =\frac{\vec{r}_{1}(s)+\vec{r}_{2}(s)}{2} \\
\vec{r}(s) & =\vec{r}_{1}(s)-\vec{r}_{2}(s)
\end{aligned}
$$

and performing the change of variable in (1), we have

$$
Z=\int \mathcal{D} \vec{r}(s) \exp \left(-\frac{d}{4 a^{2}} \int_{0}^{N} d s\left(\frac{d \vec{r}}{d s}\right)^{2}-\beta \int_{0}^{N} d s v(\vec{r}(s))-W(\{\vec{r}(s)\})\right)
$$

where

$e^{-W(\{\vec{r}(s)\})}=\int \mathcal{D} \vec{R}(s) \exp \left(-\frac{d}{a^{2}} \int_{0}^{N} d s\left(\frac{d \vec{R}}{d s}\right)^{2}-g \int_{0}^{N} d s \int_{0}^{N} d s^{\prime} \delta\left(\vec{R}(s)-\vec{R}\left(s^{\prime}\right)+\frac{\vec{r}(s)+\vec{r}\left(s^{\prime}\right)}{2}\right)\right)$

In the denatured phase, the two chains are far apart and interact very weakly; it is therefore justified to treat this interaction in a perturbative manner. Expanding the effective potential $W$ to first order in $g$, we get 


$$
e^{-W(\{\vec{r}(s)\})} \simeq \exp \left(-g \int_{0}^{N} d s \int_{0}^{N} d s^{\prime}\left\langle\delta\left(\vec{R}(s)-\vec{R}\left(s^{\prime}\right)+\frac{1}{2}\left(\vec{r}(s)+\vec{r}\left(s^{\prime}\right)\right)\right)\right\rangle_{0}\right)
$$

where the brackets stand for

$$
\langle A\rangle_{0}=\frac{1}{V} \int \mathcal{D} \vec{R}(s) A \exp \left(-\frac{d}{a^{2}} \int_{0}^{N} d s\left(\frac{d \vec{R}}{d s}\right)^{2}\right)
$$

and $V$ is the volume of the system.

We obtain

$$
W(\{\vec{r}(s)\}) \simeq g \int_{0}^{N} d s \int_{0}^{N} d s^{\prime}\left(\frac{d}{\pi\left|s-s^{\prime}\right| a^{2}}\right)^{d / 2} \exp \left(-\frac{d\left(\vec{r}(s)+\vec{r}\left(s^{\prime}\right)\right)^{2}}{4 a^{2}\left|s-s^{\prime}\right|}\right)
$$

which is valid for dimensions $d>2$. This expression is asymptotically exact in the denatured phase, where very few monomers of the two chains come close together.

Setting $S=\left(s+s^{\prime}\right) / 2$ and $\sigma=s-s^{\prime}$ in equation (8), we get for large $N$

$$
W(\{\vec{r}(s)\}) \simeq g \int_{0}^{N} d S \int_{-\infty}^{+\infty} d \sigma\left(\frac{d}{\pi|\sigma| a^{2}}\right)^{d / 2} \exp \left(-\frac{d(\vec{r}(S+\sigma / 2)+\vec{r}(S-\sigma / 2))^{2}}{4 a^{2}|\sigma|}\right)
$$

A simple scaling argument shows that this effective interaction must vanish at large relative separation. The range of $|\sigma|$ which contributes to the integral is given by $|\sigma|_{\text {typ }} \sim$ $d(\vec{r}(S+\sigma / 2)+\vec{r}(S-\sigma / 2))^{2} / 4 a^{2}$. Thus, for small separations where the interaction is sizeable, only small $\sigma$ contribute significantly to the integral and we may expand $\vec{r}(S+$ $\sigma / 2)+\vec{r}(S-\sigma / 2)$ in powers of $\sigma$, and obtain

$$
\begin{aligned}
W(\{\vec{r}(s)\}) & \simeq g \int_{0}^{N} d S \int_{-\infty}^{+\infty} d \sigma\left(\frac{d}{\pi|\sigma| a^{2}}\right)^{d / 2} \exp \left(-\frac{d \vec{r}^{2}(S)}{a^{2}|\sigma|}\right) \\
& \simeq \int_{0}^{N} d s \frac{\alpha_{d}}{|\vec{r}(s)|^{d-2}}
\end{aligned}
$$

where

$$
\alpha_{d}=2 g\left(\frac{1}{\pi}\right)^{d / 2} \Gamma\left(\frac{d}{2}-1\right) \frac{d}{a^{2}}
$$

We see that the effect of the inter-chain excluded volume interaction is to generate a "Coulomb"-like repulsive term in the relative coordinate. This entropic repulsion is familiar 
in other interacting lines problems [2,10]. Its long range nature is due to the large lateral excursions of the chains.

An alternative derivation of this term can be obtained from equation (11). The use of the identity

$$
\delta\left(\vec{r}_{1}(s)-\vec{r}_{2}\left(s^{\prime}\right)\right)=\int \frac{d^{d} \vec{k}}{(2 \pi)^{d}} e^{i \vec{k} \cdot\left(\vec{r}_{1}(s)-\vec{r}_{2}\left(s^{\prime}\right)\right)}
$$

allows us to rewrite the excluded volume term of equation (11) as

$$
\frac{g}{2} \int_{0}^{N} d s \int_{0}^{N} d s^{\prime} \int \frac{d^{d} \vec{k}}{(2 \pi)^{d}}\left(e^{i \vec{k} \cdot\left(\left(\vec{r}_{1}(s)-\vec{r}_{1}\left(s^{\prime}\right)\right)+\left(\vec{r}_{1}\left(s^{\prime}\right)-\vec{r}_{2}\left(s^{\prime}\right)\right)\right)}+e^{i \vec{k} \cdot\left(\left(\vec{r}_{1}(s)-\vec{r}_{2}(s)+\left(\vec{r}_{2}(s)\right)-\vec{r}_{2}\left(s^{\prime}\right)\right)\right)}\right)
$$

where we have explicitly introduced, in a symmetric way, the relative coordinate of the chains $\left(\vec{r}_{1}(s)-\vec{r}_{2}(s)\right)$.

Each term of equation (13) is a product over single chain and relative coordinate conformations. For each contact of the chains, it seems natural to average the single chain term. Defining this average by $G\left(\vec{k}, s, s^{\prime}\right)=\left\langle e^{i \vec{k} \cdot\left(\vec{r}(s)-\vec{r}\left(s^{\prime}\right)\right)}\right\rangle$, the excluded volume term reads

$$
g \int_{0}^{N} d s \int_{0}^{N} d s^{\prime} \int \frac{d^{d} \vec{k}}{(2 \pi)^{d}} G\left(\vec{k}, s, s^{\prime}\right) e^{i \vec{k} \cdot\left(\vec{r}_{1}(s)-\vec{r}_{2}(s)\right)}
$$

Since we have neglected the intrachain excluded volume, we have

$$
G\left(\vec{k}, s, s^{\prime}\right)=e^{-\frac{\vec{k}^{2} a^{2}}{2 d}\left|s-s^{\prime}\right|}
$$

Putting everything together, we get back equations (10,11).

We thus can write the partition function (雨) for the relative coordinate of the two chains as

$$
Z=\int \mathcal{D} \vec{r}(s) \exp \left(-\frac{d}{4 a^{2}} \int_{0}^{N} d s\left(\frac{d \vec{r}}{d s}\right)^{2}-\beta \int_{0}^{N} d s v(\vec{r}(s))-\int_{0}^{N} d s \frac{\alpha_{d}}{|\vec{r}(s)|^{d-2}}\right)
$$

For long chains, we may now use a well known quantum mechanical analogy [7] and study the ground state of the Hamiltonian

$$
H=-\frac{a^{2}}{d} \vec{\nabla}^{2}+\beta v(\vec{r})+\frac{\alpha_{d}}{|\vec{r}|^{d-2}}
$$


This ground state (wavefunction $\Psi_{0}$, energy $E_{0}$ ) is given by the Schrödinger equation

$$
H \Psi_{0}=E_{0} \Psi_{0}
$$

The free energy per monomer then reads $F=E_{0} T$.

We now discuss this equation as a function of space dimensionality.

- $2<d<4$

We discuss the typical case $d=3$. The potential is radial, and thus the ground state will be spherically symmetric.

In radial coordinates, equation (18) becomes

$$
-\frac{a^{2}}{3} u_{0}^{\prime \prime}+\beta v(r) u_{0}+\frac{\alpha_{3}}{r} u_{0}=E_{0} u_{0}
$$

where $u_{0}(r)=r \Psi_{0}(r)$ and $\alpha_{3}$ is given by (11).

The unbinding transition [8,9 occurs when $T=T_{c}$ and $E_{0}=0$. As the binding potential vanishes for $r>r_{0}$, we have in this region

$$
-\frac{a^{2}}{3} u_{0}^{\prime \prime}+\frac{\alpha_{3}}{r} u_{0}=0
$$

The solution of this equation which vanishes at infinity is given by a Bessel function

$$
u_{0}(r)=\sqrt{\frac{r}{\lambda}} K_{1}\left(\sqrt{\frac{r}{\lambda}}\right)
$$

with

$$
u_{0}(r) \simeq\left(\frac{r}{\lambda}\right)^{1 / 4} \exp \left(-\sqrt{\frac{r}{\lambda}}\right) \text { for } r \rightarrow \infty
$$

The (finite) correlation length $\lambda$ is given by

$$
\lambda=\frac{a^{2}}{12 \alpha_{3}}=\frac{\pi a^{4}}{72 g}
$$

implying that the transition is first-order. This is due to the strongly repulsive barrier generated by the excluded-volume. The transition temperature $T_{c}$ is obtained by 
matching the above solution to the solution inside the attractive region $\left(v(r)=-V_{0}\right.$ for $r<r_{0}$ ). The calculations are rather tedious and will not be given here. It is however interesting to derive an estimate of the latent heat at the transition. We therefore rewrite equation (19) as

$$
-\frac{a^{2}}{3} u_{0}^{\prime \prime}+\beta_{c} v(r) u_{0}+\left(\beta-\beta_{c}\right) v(r) u_{0}+\frac{\alpha_{3}}{r} u_{0}=E_{0} u_{0}
$$

Close to $T_{c}$, we do first order perturbation theory and get

$$
E_{0}=\left\langle u_{0 c}(r)\left|\left(\beta-\beta_{c}\right) v(r)\right| u_{0 c}(r)\right\rangle
$$

where $u_{0 c}(r)$ denotes the full solution of equation (19) at the critical temperature. Since $F \simeq T_{c} E_{0}$, we finally obtain for the latent heat $L\left(L=T_{c}\left(\frac{\partial F}{\partial T}\right)_{T_{c}}\right)$

$$
L=-\left\langle u_{0 c}(r)|v(r)| u_{0 c}(r)\right\rangle
$$

A simpler procedure [7] replaces the attractive region by an attractive $\delta$-potential of strength $\gamma$ at $r=r_{0}$ leading to

$$
\begin{aligned}
F & =-\gamma\left(\frac{T_{c}-T}{T}\right) u_{0}^{2}\left(r_{0}\right) \text { for } T \rightarrow T_{c}^{-} \\
& =0 \text { for } T>T_{c}
\end{aligned}
$$

and a latent heat $L=\gamma u_{0}^{2}\left(r_{0}\right)$, where $u_{0}\left(r_{0}\right)$ is given in equation (21).

- $d>4$

The ground state $\Psi_{0}$ satisfies the radial equation

$$
-\frac{a^{2}}{d}\left(\frac{d^{2}}{d r^{2}}+\frac{d-1}{r} \frac{d}{d r}\right) \Psi_{0}+\left(\beta v(r)+\frac{\alpha_{d}}{r^{d-2}}\right) \Psi_{0}=E_{0} \Psi_{0}
$$

The unbinding point occurs for $E_{0}=0$. It is easily seen that at large distances, the "Coulomb" potential is negligible, and thus the wave-function decays as a power-law 


$$
\Psi_{0}(r) \sim \frac{1}{r^{d-2}} \text { for } r \rightarrow \infty
$$

This wave-function is normalizable for $d>4$, and thus, the transition is again firstorder. This is in agreement with the fact that in $d>4$, the excluded-volume effect should be irrelevant and thus one should recover the results of the Gaussian adsorption problem. According to the Poland-Scheraga theory [1], the denaturation transition is first-order if the exponent $\theta$ describing the probability of first return to the origin of the chain is larger than 2. For a Gaussian chain in $d>4$, this is indeed the case since $\theta=\frac{d}{2}$.

However, this wave-function is much less localized than below four dimensions: only moments of $r$ of order strictly smaller than $(d-4)$ are finite.

- $d=4$

This case is marginal, since the Laplacian operator and the "Coulomb" potential have the same scaling dimension. At large distance, the wave-function satisfies

$$
-\frac{a^{2}}{4}\left(\frac{d^{2}}{d r^{2}}+\frac{3}{r} \frac{d}{d r}\right) \Psi_{0}+\frac{\alpha_{4}}{r^{2}} \Psi_{0}=0
$$

The wave-function behaves as a power-law

$$
\Psi_{0} \sim \frac{1}{r^{\nu}}
$$

with

$$
\nu=1+\sqrt{1+\frac{4 \alpha_{4}}{a^{2}}}
$$

The wave-function is normalizable, but again with an infinite correlation length and only a finite number of moments of $r$ are defined.

We have studied a simple model for the denaturation of DNA, where we have given an asymptotically exact treatment of the interchain excluded volume interaction. We find that the first order character of the denaturation transition stems from the existence of 
this long range interaction, in broad agreement with recent work [5,6]. The difference in our results might be due to our neglect of the intrachain excluded volume. Within their scaling approximation, the latter authors find that the first order transition is associated with a large loop probability distribution, whereas our approximation yields an exponentially bound state for $d=3$. Future work is needed to clarify this point. Sequence heterogeneity [11, and chain stiffness 12,13 raise further questions.

\section{ACKNOWLEDGEMENTS}

We would like to thank Y.Kafri and D.Mukamel for many useful discussions. 


\section{REFERENCES}

[1] D. Poland and H. Scheraga, Theory of Helix-Coil transition in Biopolymers, Academic Press, New York (1970) and references therein.

[2] M.E. Fisher, J. Stat. Phys. 34, 667 (1984).

[3] T. Dauxois and M. Peyrard, Phys. Rev. E, 51, 4027 (1995).

[4] S. Cocco and R. Monasson, Phys. Rev. Lett. 83, 5178 (1999).

[5] M. S. Causo, B. Coluzzi and P. Grassberger, Phys. Rev E, 62, 3958 (2000).

[6] Y. Kafri, D. Mukamel and L. Peliti, Phys. Rev. Lett. 85, 4988 (2000).

[7] P.G.de Gennes, Scaling concepts in polymer physics, Cornell University Press, Ithaca, (1979).

[8] R.K.P. Zia, R. Lipowsky and D.M. Kroll, Amer. J. Phys., 56, 160 (1988).

[9] G. Gompper, in Biologically Inspired Physics, p. 175, L. Peliti (Ed.), Plenum Press, New York (1991) and references therein.

[10] V.L. Pokrovsky and A.L. Talapov, Phys. Rev. Lett. 42, 65 (1979).

[11] D. Cule and T. Hwa, Phys. Rev. Lett. 79, 2375 (1997).

[12] N. Theodorakopoulos, T. Dauxois and M. Peyrard, Phys. Rev. Lett., 85, 6, (2000).

[13] R. Bundschuh, M. Lassig and R. Lipowsky, Eur. Phys. J. E, 3, 295 (2000). 